\title{
COMPARAÇÃO ENTRE DIFERENTES TRATAMENTOS QUÍMICOS APLICADOS ÀS FOLHAS DE BANANEIRA PARA ISOLAMENTO DA CELULOSE
}

\author{
E. P. SANTOS ${ }^{1 *}$, M. C. A. NONO ${ }^{1}$ e M. L. C. P. da SILVA ${ }^{2}$ \\ ${ }^{1}$ Instituto Nacional de Pesquisas Espaciais, Laboratório Associado de Sensores e Materiais \\ ${ }^{2}$ Universidade de São Paulo, Departamento de Engenharia Química \\ E-mail para contato: esterzinha.psantos@gmail.com
}

\begin{abstract}
RESUMO - Os resíduos agrícolas, provenientes de fibras naturais e ricos em celulose, são um grande atrativo na produção de novos materiais, pois possuem baixo custo, baixa densidade, alta disponibilidade, são renováveis e atóxicos. Visando o aumento da adesão na interface fibra/matriz, as modificações químicas apresentam-se como alternativa no favorecimento da interação entre os componentes para aplicação como reforço em matrizes poliméricas. $\mathrm{O}$ objetivo do trabalho é avaliar qual a melhor proposta de tratamento para o isolamento da celulose. Para tal, foram avaliadas três metodologias de ataque químico: ataque com $\mathrm{CH}_{3} \mathrm{COOH} / \mathrm{NaOH}$, dois ataques com $\mathrm{CH}_{3} \mathrm{COOH}$ e a última com dois ataques ácidos/NaOH. A celulose isolada foi caracterizada por FTIR, TGA/DTG, DRX e testes de intumescimento em água. Os resultados de DRX e FTIR apresentaram-se similares em todas as propostas. As curvas TGA/DTG e o teste de intumescimento em água, possibilitaram definir as condições de tratamento para isolamento da celulose.
\end{abstract}

\section{INTRODUÇÃO}

Fibras naturais são suscetíveis a modificações químicas, devido à presença de grupos hidroxila, os quais podem ligar-se a outras moléculas com uma melhora nas características superficiais das fibras, tais como adesão, molhabilidade, tensão superficial ou porosidade (OTTOBONI, 2011). Além disso, essas modificações químicas apresentam-se como alternativa no favorecimento da interação entre os componentes para aplicação como reforço em matrizes poliméricas (SANCHEZ et al., 2010).

Os tratamentos químicos podem ser definidos como aqueles em que empregam-se substâncias químicas, as quais irão reagir com a fibra, favorecendo uma maior interação entre os constituintes do compósito. As ligações podem ser: covalente, ligação de hidrogênio ou ácido-base (ALBINANTE et al., 2013).

Diversos tratamentos químicos são empregados na modificação química das fibras, tais como ácido acético, silano, ácido acrílico, isocianatos, permanganato de potássio, peróxidos, dentre outros (LI et al., 2007). Dentre estes tratamentos, o tratamento alcalino (mercerização) é uma das modificações químicas mais baratas e utilizadas nas fibras naturais, quando as mesmas são empregadas como reforço (SANCHEZ et al., 2010). Porém, para minimizar as agressões ambientais destes componentes tóxicos, outros tipos de tratamentos químicos estão 
sendo estudados, como por exemplo, a utilização de uma mistura ácida de $\mathrm{HNO}_{3} / \mathrm{CH}_{3} \mathrm{COOH}$ (SILVA, 2013; BRENDEL et al., 2000).

Neste contexto, este trabalho tem como objetivo avaliar qual a melhor proposta de tratamento para o isolamento da celulose e verificar a influência destas metodologias nas propriedades físico-químicas da celulose.

\section{MATERIAIS E MÉTODOS}

\subsection{Materiais}

As folhas de bananeira (Musa sapientum) empregadas nos tratamentos químicos foram previamente lavadas, secas a temperatura ambiente e trituradas. Para o ataque ácido utilizouse uma mistura ácida de ácido acético, $\mathrm{CH}_{3} \mathrm{COOH}, 80 \% \mathrm{v} / \mathrm{v}$ e ácido nítrico, $\mathrm{HNO}_{3}, 65 \% \mathrm{~m} / \mathrm{m}$. E para o ataque básico uma solução de hidróxido de sódio, $\mathrm{NaOH}, 1,5 \mathrm{~mol} / \mathrm{L}$.

\subsection{Isolamento da celulose}

Para verificar qual a melhor proposta de tratamento químico para o isolamento da celulose foram avaliadas três propostas diferentes, de acordo com a Tabela 1.

Tabela 1 - Tratamentos químicos empregados para o isolamento da celulose

\begin{tabular}{|c|c|}
\hline METODOLOGIA & TRATAMENTO QUíMICO \\
\hline Proposta 1 (P1) & 1 ataque ácido + 1 ataque básico \\
\hline Proposta 2 (P2) & 2 ataques ácidos \\
\hline Proposta 3 (P3) & 2 ataques ácidos + 1 ataque básico \\
\hline
\end{tabular}

Ataque ácido: para este tratamento empregou-se uma adaptação da metodologia proposta por Brendel et al. (2000). As folhas de bananeira previamente secas e trituradas, foram transferidas para um erlenmeyer e adicionada uma mistura de ácidos acético/nítrico. $\mathrm{O}$ sistema foi mantido sob agitação a $120{ }^{\circ} \mathrm{C}$ por 20 minutos e, após aquecimento, filtrado a vácuo, lavado com água deionizada até $\mathrm{pH}$ neutro e com etanol para a remoção de resíduos orgânicos.

Ataque básico: a celulose isolada, proveniente do tratamento ácido foi transferida para um béquer, imersa em solução de $\mathrm{NaOH} 1,5 \mathrm{~mol} / \mathrm{L}$ e mantida sob aquecimento a $40-50^{\circ} \mathrm{C}$ por 30 minutos. Em seguida, foi filtrada a vácuo, lavada com água deionizada até pH neutro e com etanol para a remoção de resíduos orgânicos. As celuloses obtidas ao final de cada proposta foram secas em estufa a $50{ }^{\circ} \mathrm{C}$ até peso constante.

\subsection{Caracterizações}

A fibra bruta e a celulose obtida em cada proposta foram caracterizadas por 
espectroscopia na região do infravermelho com transformada de Fourier (FTIR), termogravimetria e sua derivada (TGA/DTG), difração de raios X (DRX) e testes de intumescimento.

A presença de grupos funcionais ligados à estrutura da folha de bananeira e à celulose, foi determinada por FTIR, Perkin Elmer, modelo Spectrum GX, na faixa de comprimento de onda de 400 a $4000 \mathrm{~cm}^{-1}$.

A cristalinidade da folha de bananeira e das diferentes celuloses foi determinada por DRX, em um difratômetro PANalytical, modelo Empyrean, com fonte de radiação CuKa, tensão de $40 \mathrm{kV}$ e corrente de $30 \mathrm{~mA}$, para valores de $2 \theta$ entre 10 e $90^{\circ}$.

O comportamento térmico de todos os materiais foi avaliado por TGA, em uma termobalança Shimadzu, modelo TGA-50, sob atmosfera de nitrogênio, com vazão de 50 $\mathrm{mL} / \mathrm{min}$ e taxa de aquecimento de $20^{\circ} \mathrm{C} / \mathrm{min}$, no intervalo da temperatura ambiente até $900^{\circ} \mathrm{C}$.

Realizou-se testes de intumescimento em água com o objetivo de verificar a dispersão da celulose em água. Manteve-se a celulose imersa por 1 hora e realizou-se uma avaliação macroscópica.

\section{RESULTADOS E DISCUSSÃO}

\subsection{Espectroscopia na região do infravermelho com transformada de Fourier, FTIR}

A Figura 1 apresenta os espectros de infravermelho para a fibra bruta e para as três propostas de tratamento químico.

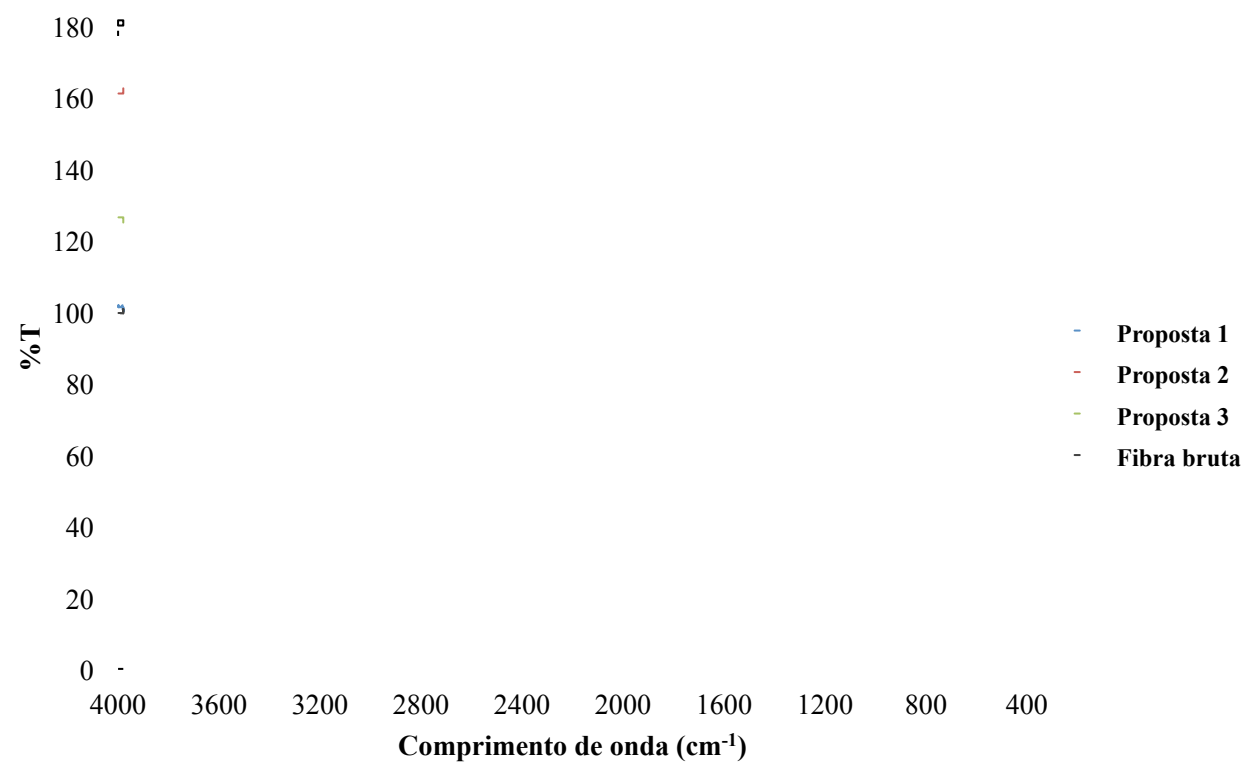


Figura 1 - Espectro de absorção na região do infravermelho para fibra bruta e celulose isolada a partir das diferentes metodologias de tratamento químico.

Comparando-se os espectros de infravermelho para a fibra bruta e os três tratamentos químicos observou-se comportamento similar em todos os casos, o que impossibilitou a determinação da metodologia ideal para isolamento da celulose. Para esta avaliação o FTIR não se mostra uma metodologia eficaz.

\subsection{Difração de raios $X, D R X$}

A Figura 2 compara os difratogramas para a fibra bruta e celuloses isoladas obtidas por diferentes metodologias.

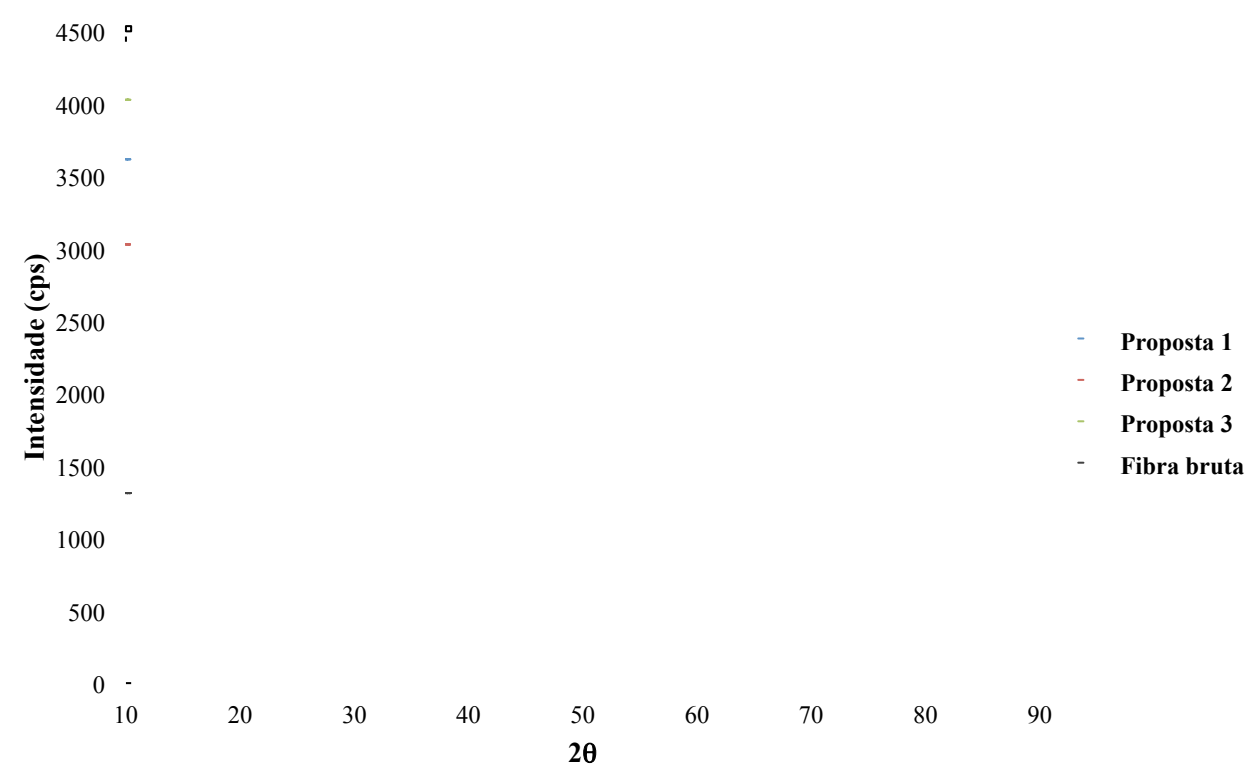

Figura 2 - Difratogramas de raios X para fibra bruta e celulose isolada a partir das diferentes metodologias de tratamento químico.

Para as três propostas, os difratogramas da celulose apresentam três picos característicos com os valores de $2 \theta=15^{\circ}, 22^{\circ}$ e $35^{\circ}$, os quais correspondem aos planos cristalográficos (101), (002) e (040), respectivamente. Segundo Guimarães et al. (2010), estes picos característicos são intrínsecos de fibras lignocelulósicas. De acordo com Ass et al. (2006), a celulose tipo I exibe um pico referente à fração cristalina em $22^{\circ} \leq 2 \theta \leq 23^{\circ}$, enquanto para a celulose tipo II este pico ocorre em $18^{\circ} \leq 2 \theta \leq 22^{\circ}$. As regiões correspondentes à fração amorfa para a celulose tipo I e celulose tipo II ocorrem em $18^{\circ} \leq 2 \theta \leq 19^{\circ}$ e $13^{\circ} \leq 2 \theta \leq 15^{\circ}$, respectivamente. A celulose obtida em todos as propostas foi classificada como celulose tipo I.

A partir dos resultados obtidos nos difratogramas foi possível estimar o índice de cristalinidade $\left(I_{c}\right)$ do material celulósico, definido pela Equação 1 (BUSCHLE-DILLER e ZERONIAN, 1992; GUIMARÃES et al., 2009): 


$$
I_{c}=1-\frac{I_{M I N}}{I_{M A ́ X}} \times 100
$$

$\mathrm{I}_{\mathrm{MIN}}=$ intensidade mínimo do vale, referente ao material amorfo, na região de $2 \theta=18^{\circ}$;

$\mathrm{I}_{\mathrm{MÁX}}=$ intensidade máxima do pico de difração, referente ao material cristalino, na região de $2 \theta=22^{\circ}$.

A Tabela 2 apresenta os valores dos $I_{c}$ para a fibra bruta e celulose isolada a partir dos diferentes tratamentos químicos.

Tabela 2 - Valores dos índices de cristalinidade $\left(I_{c}\right)$

\begin{tabular}{|c|c|c|c|}
\hline Material & $\mathbf{I}_{(\mathbf{0 0 2})}$ & $\mathbf{I}_{\mathbf{a m}}$ & $\mathbf{I}_{\mathbf{c}} \mathbf{( \% )}$ \\
\hline Fibra bruta & 1344,8 & 956,5 & 28,9 \\
\hline Celulose (P1) & 3652,3 & 1258 & 65,6 \\
\hline Celulose (P2) & 3034,2 & 1148 & 62,2 \\
\hline Celulose (P3) & 4010,2 & 1343 & 66,5 \\
\hline
\end{tabular}

De acordo com a Tabela 2, houve o aumento significativo do índice de cristalinidade, comparado à fibra de bananeira bruta pela eliminação da lignina e hemicelulose (frações amorfas) presentes, confirmando o isolamento da celulose de maneira satisfatória pelas três metodologias propostas (PEREIRA et al., 2014). A similaridade dos resultados entre as três propostas indicam que a técnica de DRX também se mostrou insatisfatória para a definição da metodologia.

\subsection{Termogravimetria, TGA/DTG}

A Tabela 3 apresenta os dados das curvas TGA/DTG da fibra de bananeira bruta e da celulose isolada, a partir das três propostas, em atmosfera de nitrogênio. 
Tabela 3 - Dados das curvas TGA/DTG para a fibra bruta e celulose isolada a partir das diferentes propostas

\begin{tabular}{|c|c|c|c|c|}
\hline MATERIAL & $\begin{array}{c}\text { Intervalos } \\
\left({ }^{\circ} \mathrm{C}\right)\end{array}$ & $\begin{array}{l}\text { Perda de } \\
\text { massa }(\%)\end{array}$ & $\begin{array}{l}\text { Temp. Pela } \\
\text { DTG }\left({ }^{\circ} \mathrm{C}\right)\end{array}$ & $\begin{array}{c}\text { Resíduo } \\
(\%)\end{array}$ \\
\hline FIBRA BRUTA & $\begin{array}{c}25-166 \\
166-439 \\
439-527 \\
527-900\end{array}$ & $\begin{array}{c}7,83 \\
46,52 \\
19,49 \\
14,28\end{array}$ & $\begin{array}{c}78 \\
334 \\
507 \\
-\end{array}$ & 11,88 \\
\hline CELULOSE P1 & $\begin{array}{c}25-167 \\
167-392 \\
392-900\end{array}$ & $\begin{array}{c}4,45 \\
65,15 \\
22,61\end{array}$ & $\begin{array}{c}68 \\
353 \\
414\end{array}$ & 7,79 \\
\hline CELULOSE P2 & $\begin{array}{c}25-179 \\
179-471 \\
471-900\end{array}$ & $\begin{array}{c}4,63 \\
71,18 \\
15,37\end{array}$ & $\begin{array}{c}69 \\
362 \\
564\end{array}$ & 8,82 \\
\hline CELULOSE P3 & $\begin{array}{c}25-175 \\
175-400 \\
400-900\end{array}$ & $\begin{array}{c}4,25 \\
70,02 \\
20,41\end{array}$ & $\begin{array}{c}67 \\
355 \\
415\end{array}$ & 5,32 \\
\hline
\end{tabular}

Segundo a Tabela 3 todas as curvas TGA/DTG para a celulose isolada a partir das propostas apresentam três eventos térmicos, os quais são referentes à desidratação, à decomposição e à degradação, respectivamente. A proposta 2 apresenta maior perda de massa $(71,18 \%)$ no evento de decomposição e com menor perda de massa no evento de degradação $(15,37 \%)$. Esta diferença pode estar relacionada ao tratamento químico empregado (dois ataques ácidos), pois a mistura de ácidos promove uma maior degradação preferencialmente nas regiões amorfas, preservando a fração cristalina (CAO e TAN, 2002). Outro efeito observado foi a alteração na temperatura de degradação, com maior temperatura para a proposta $2\left(362{ }^{\circ} \mathrm{C}\right)$, quando comparada à fibra bruta $\left(334^{\circ} \mathrm{C}\right)$. Isso pode estar associado à adsorção de grupos acetila em substituição à hidroxila em maior número de sítios ativos, $\mathrm{o}$ que protege a superfície e estabiliza o material. Em decorrência disso, observa-se um maior valor residual para a P2 caracterizando um material com maior estabilidade térmica (LOPES et al., 2011; PEREIRA et al., 2011). A Figura 3 apresenta os perfis das curvas TGA/DTG para todos os materiais avaliados. 


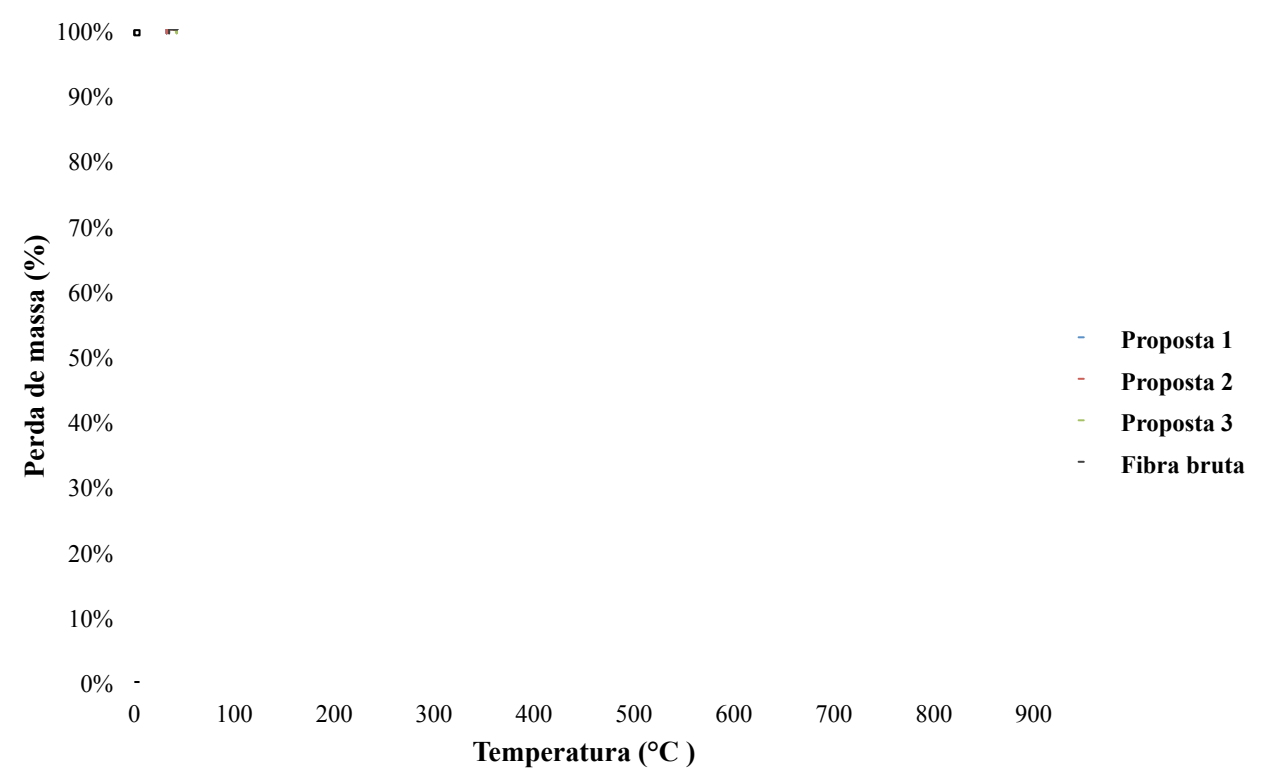

Figura 3 - Curvas TGA/DTG para fibra bruta e celulose isolada a partir das diferentes metodologias de tratamento químico.

\subsection{Testes de intumescimento em água}

As celuloses obtidas nos tratamentos químicos foram submetidas a testes de intumescimento em água por $1 \mathrm{~h}$. Segundo os testes, somente a celulose obtida na proposta 2 dispersou-se em água, com a liberação das fibras curtas de celulose geradas, proporcionando um aumento na área de contato, o que não ocorreu nas outras duas propostas. Este aumento é interessante pois favorecerá a adição de óxidos metálicos hidratados para a obtenção de novos materiais híbridos orgânico-inorgânico.

\section{CONCLUSÃO}

As técnicas de DRX e FTIR não foram conclusivas na definição da melhor proposta, pois seus resultados apresentaram-se similares.

As curvas TGA/DTG para as três propostas apresentaram um aumento na temperatura de decomposição, comparada à fibra bruta, porém a proposta 2 apresentou uma maior diferença nesta temperatura, caracterizando o aumento da estabilidade térmica da celulose, característica crucial para sua definição como melhor proposta.

O teste de intumescimento em água foi decisivo para a escolha do tratamento, pois somente na celulose da proposta 2 houve dispersão de suas fibras curtas no meio.

\section{REFERÊNCIAS}

ALBINANTE, S. R. et al. Revisão dos tratamentos químicos da fibra natural para mistura com poliolefina. Quim. Nova, v. 36, n. 1, 114-122, 2013. 
ASS, B. A. P. et al. Mercerized linters cellulose: characterization and acetylation in N,Ndimethylacetamide/lithium chloride. Carbohydr. Polym., v. 63, p. 19-29, 2006.

BRENDEL, O. et al. A rapid and simple method to isolate pure alpha-cellulose. Phytochem. Anal., v. 11, p. 7-10, 2000.

BUSCHLE-DILLER, G.; ZERONIAN, S. H. Enhancing the reactivity and strength of cotton fibers. J. Appl. Polym. Sci., v. 45, p. 967-979, 1992.

CAO, Y.; TAN, H. Effects of cellulase on the modification of cellulose. Carbohydr. Res., v. 337, p. 1291-1296, 2002.

GUIMARÃES, J. L. et al. Characterization of banana, sugarcane bagasse and sponge gourd fibres of Brazil. Ind. Crop. Prod., v. 30, p. 407-415, 2009.

GUIMARÃES, J. L. et al. Studies of the processing and characterization of corn starch and its composites with banana and sugarcane fibers from Brazil. Carbohydr. Polym., v. 80, p. 130$138,2010$.

LI, X. et al. Chemical treatments of natural fiber for use in natural fiber-reinforced composites: a review. J. Polym. Environ., v.15, p. 25-33, 2007.

LOPES, F. F. M. et al. Modificação das propriedades das fibras de curauá por acetilação. Rev. Bras. Eng. Agri. Amb., v. 15, n. 3, p. 316-321.

OTTOBONI, F. S. Desenvolvimento de novos compósitos baseados em polietileno e híbridos gerados a partir de resíduos agrícolas modificados com óxido de nióbio. 121f. Dissertação de Mestrado, Universidade de São Paulo, São Paulo, 2011.

PEREIRA, P. H. F. et al. Sugarcane bagasse pulping and bleaching: thermal and chemical characterization. BioRes., v. 6, n. 3, p. 2471-2482, 2011.

PEREIRA, P. H. F. et al. Sugarcane bagasse cellulose fibres and their hydrous niobium phosphate composites: synthesis and characterization by XPS, XRD and SEM. Cellulose, v. 21, p. 641-652, 2014.

SANCHEZ, E. M. S. et al. Compósito de resina de poliéster insaturado com bagaço de canade-açúcar: influência do tratamento das fibras nas propriedades. Polím., v. 20, n. 3, p. 194200, 2010. 